\title{
Variables que caracterizan los gráficos estadísticos y las tareas relacionadas con ellos en los libros de texto de educación secundaria en Costa Rica
}

\author{
Pedro Arteaga, Universidad de Granada (España) \\ Maynor Jiménez-Castro, Universidad de Costa Rica (Costa Rica) \\ Carmen Batanero, Universidad de Granada (España)
}

\begin{abstract}
Variables que caracterizan los gráficos estadísticos y las tareas relacionadas con ellos en los libros de texto de educación secundaria en Costa Rica

\section{Resumen}

El objetivo de la investigación fue analizar las variables que caracterizan los gráficos estadísticos y las tareas relacionadas en los libros de texto de educación secundaria en Costa Rica. Primero se examinan 143 gráficos presentados en tres editoriales para los cursos 7. ${ }^{\circ}$ a 9. ${ }^{\circ}$ (de 13 a 15 años), estudiando el tipo de gráfico, su complejidad semiótica y el contexto de los datos representados. Seguidamente se estudian 340 tareas propuestas sobre estos gráficos, analizando el nivel de lectura requerido, propósito del gráfico dentro de la tarea y tipo de tarea. Los resultados se comparan con otros estudios de gráficos estadísticos en libros de texto de educación primaria. En comparación con la anterior etapa educativa se introducen nuevos gráficos, hay mayor presencia del nivel superior de complejidad semiótica y disminuye el contexto escolar, dando mayor peso al científico. Respecto a las tareas, disminuye el peso de la lectura, apareciendo nuevas tareas; el propósito principal es el análisis y no hay mucha diferencia en el nivel de lectura requerido en primaria y secundaria.
\end{abstract}

Palabras clave. Gráficos estadísticos; libro de texto; educación primaria; Costa Rica; educación matemática.

Variables characterizing statistical graphs and related tasks in Costa Rica secondary education textbooks

\section{Abstract}

The aim of this research was to analyze the variables characterizing the statistical graphs and related tasks in Costa Rica secondary education textbooks. We first examine 143 graphs presented in three editorials for grades 7 to 9 (from 13 to 15 years old), and study the type of graph, its semiotic complexity, and the context of the data represented. We then study 340 tasks related to these graphs, by analyzing the reading level required, purpose of the graph within the task and type of task. We compare the results with other statistical graph research carried out in primary education textbooks. Compared to the previous educational stage, new graphics are introduced; there is a greater presence of the higher level of semiotic complexity and the school context decreases, giving more weight to the scientific one. Regarding the tasks, the weight of reading decreases, with new tasks appearing, the main purpose is analysis and there is not much difference in the reading level required in primary and secondary education.

Keywords. Statistical graphs; textbook; primary education; Costa Rica; mathematics education.

\section{Introducción}

Dada la variedad y cantidad de gráficos estadísticos en los medios de comunicación y el trabajo profesional, el ciudadano debe adquirir una cultura estadística mínima que permita una interpretación crítica (Engel, 2019; Weiland, 2017). Un elemento clave en la cultura estadística es el conocimiento de los gráficos estadísticos, que con frecuencia aparecen en los medios de comunicación (Roberts y Brugar, 2017), aunque dicha comprensión no siempre se alcanza en los estudiantes (Whitaker y Jacobbe, 2017). La nece- 
sidad de mejorar el conocimiento de los estudiantes ha llevado al Ministerio de Educación Pública de Costa Rica a fortalecer la enseñanza de los gráficos estadísticos en sus programas de matemáticas (MEP, 2012). En los cursos séptimo a noveno (13 a 15 años) en que se centra el trabajo, se proponen respectivamente estos contenidos:

- Se tratan conceptos básicos de estadística, recolección de información, representación y caracterización de datos con moda, media, mediana y recorrido.

- Se fomenta la recolección de datos y su representación en gráficos de barras, sectores, lineales y diagramas de puntos con frecuencias absolutas o relativas. Además, se utilizan los gráficos como apoyo en el estudio de la probabilidad.

- Se deben diferenciar variables cuantitativas discretas o continuas, comprender la agrupación en intervalos y representar información con histogramas o polígonos de frecuencia. Los gráficos también surgen en el estudio de la probabilidad frecuencial.

El éxito en la formación de los alumnos requiere que el profesor conozca las características de las tareas en los libros de texto en su país y su posible dificultad, lo que permitirá mejorar la secuenciación de su enseñanza. De acuerdo con esto, el objetivo del trabajo es caracterizar los gráficos estadísticos presentados en el tercer ciclo de la educación básica (cursos de séptimo a noveno) y las tareas propuestas en relación a ellos.

Este objetivo es relevante porque no hay análisis de la presentación de gráficos estadísticos en textos de educación secundaria, donde se amplía la importancia y la variedad de gráficos estadísticos con respecto a educación primaria. Muñiz y Díaz (2018) sintetizan investigaciones sobre contenidos estadísticos en textos de bachillerato y no se centran en los gráficos. Completamos estos y otros estudios sobre contenidos estadísticos en libros de texto de secundaria como Gea et al. (2015) y del Pino y Estepa (2019). Nos centramos en el libro de texto por constituir un paso intermedio entre directrices curriculares y currículo implementado en clase (Herbel, 2007) y por su influencia en el aprendizaje del alumno (Reys et al., 2004).

\section{Fundamentos del trabajo}

Tomamos modelos teóricos sobre niveles de dificultad en la lectura de gráficos y niveles de complejidad semiótica de los gráficos, que serán variables de análisis. Además, tomamos estudios sobre gráficos estadísticos en libros de educación primaria.

\subsection{Niveles de lectura y complejidad semiótica de gráficos estadísticos}

Un gráfico es un objeto semiótico complejo, debido a los diferentes componentes que es necesario comprender para trabajar con ellos (título, escalas, tipos y cantidad de datos y elementos utilizados para representarlos). El constructor del gráfico codifica la información representada por medio de dichos elementos y el lector del gráfico debe realizar una serie de procesos interpretativos de cada uno de estos componentes y del gráfico en su conjunto (Arteaga y Batanero, 2011; Spence, 1991).

Los objetos matemáticos implícitos en un gráfico estadístico pueden ser más o menos abstractos. Teniendo en cuenta dichos objetos, así como el tipo de información representada, Arteaga y Batanero (2011) y Batanero et al. (2010) definieron niveles de complejidad semiótica de los gráficos estadísticos, que tendremos en cuenta:

S1. Representación de datos aislados. Cuando el gráfico representa unos pocos valores, por ejemplo, los datos de un solo estudiante, sin referirse a una muestra o población. No se usa la idea de variable o distribución. 
S2. Representación de un conjunto de datos sin formar su distribución. Son aquellos gráficos que representan todos los datos de una muestra o población en forma de listado, sin organizar su distribución de frecuencias ni ordenar sus valores. En esta representación se utiliza la idea de variable, valor y recorrido.

S3. Representación de la distribución de frecuencias de una variable. Si se representa una distribución, es decir, los valores de la variable y sus frecuencias. Se añade a la variable, valor y recorrido, las ideas de orden numérico, distribución y promedio.

S4. Representación de una distribución bidimensional o de mayor dimensión. Se representan varias variables a la vez; además de los objetos matemáticos anteriores, aparecen las ideas de asociación, distribución conjunta, marginal y condicional.

Por otro lado, sobre un mismo gráfico estadístico es posible plantear preguntas de diferente dificultad, lo que ha llevado a definir niveles de lectura asociadas a las tareas sobre gráficos estadísticos. En nuestro trabajo, se utilizan los siguientes, definidos por Curcio (1987), Friel et al. (2001) y Shaughnessy et al. (1996):

L1. Leer los datos. Se requiere únicamente la lectura literal de un elemento del gráfico, por ejemplo, identificar la frecuencia que corresponde a un valor de la variable.

L2. Leer entre los datos. Se precisa comparar o realizar cálculos con conjuntos de datos del gráfico, por ejemplo, determinar el mínimo.

L3. Leer más allá de los datos. Consiste en inferir una información no representada en el gráfico. Por tanto, sería necesaria una interpolación o una extrapolación.

L4. Leer detrás de los datos. Es necesario valorar críticamente el contenido del gráfico, los datos representados o las conclusiones obtenidas a partir del mismo.

\subsection{Antecedentes de nuestro trabajo}

Tomamos también investigaciones sobre gráficos estadísticos en libros de texto de educación primaria como la de Lemos (2006), que analiza tres series de libros de texto de los cursos $1 .^{\circ}$ a $4 .^{\circ}$ de educación primaria en Brasil, estudiando el tipo de gráfico y la actividad pedida. Los gráficos se reducen casi por completo al diagrama de barras y las principales actividades son el cálculo y la lectura del gráfico.

Arteaga (2011) analiza el tipo de gráfico y su nivel de complejidad semiótica, la actividad y nivel de lectura en una serie completa de libros de texto de educación primaria en España. Todas estas variables son retomadas por Díaz-Levicoy en trabajos sobre libros de texto de educación primaria con conclusiones similares: Díaz-Levicoy et al. (2016, 2017, 2018). Al estudiar tipo de gráfico, nivel de complejidad semiótica, actividad y niveles de lectura, se concluye la mayor presencia del gráfico de barras, con poco peso de otros tratados en el currículo. Los niveles de lectura más frecuentes son los intermedios en la clasificación de Curcio (1987).

Jiménez-Castro et al. (2020) es un precedente que analiza gráficos estadísticos en dos series de libros de educación primaria (cursos $1 .^{\circ}$ a $6 .^{\circ}$ ) en Costa Rica, utilizando las variables anteriores, además de contexto y propósito del gráfico. Se observaron diagramas de barras y lineales (simples y múltiples), diagrama de sectores, puntos y pictogramas, con predominio de diagrama de barras. Generalmente el nivel de lectura en las actividades es L2, 'leer entre los datos' y la complejidad semiótica del gráfico S3, 'representación de una distribución'. Las tareas más frecuentes fueron leer y calcular a partir del gráfico, contexto escolar y propósito del análisis o comunicación. 
Continuando la anterior investigación se presenta en este trabajo el análisis de los gráficos estadísticos en tres editoriales para los cursos 7. ${ }^{\circ}$ a $9 .^{\circ}$ de Educación Básica, equivalentes a los cursos $1 .^{\circ}$ a $3 .^{\circ}$ de la Educación Secundaria Obligatoria en España.

\section{Metodología}

Se trata de una investigación exploratoria, pues no hay antecedentes sobre el tema en Costa Rica. Además, es una investigación descriptiva y cualitativa que analiza documentos (libros de texto). La muestra son tres series de libros comunes en la etapa secundaria de Costa Rica, publicados en 2017, que atienden a las políticas curriculares de la reforma matemática en 2012: Publicaciones Innovadoras para Matemática en Secundaria (PI), Editorial Santillana (SA) y Publicaciones Porras (PO). El análisis de contenido permitió identificar de manera sistemática y objetiva características de un texto, a través de tres fases (Porta y Silva, 2003):

- Determinar el objeto o tema de análisis. En cada libro se estudian las actividades que incorporan gráficos estadísticos.

- Determinar las reglas de codificación y categorías. Se procede a identificar a priori, a partir de investigaciones previas, las categorías sobre tipo de gráfico, complejidad semiótica, tareas en los libros, propósitos y contexto.

- Comprobar la fiabilidad del sistema de codificación-categorización. Se revisa la codificación independientemente por los tres autores, y los pocos casos discordantes se revisan conjuntamente, hasta llegar a un acuerdo en la codificación.

En estos libros se realizaron dos tipos de análisis. El primero consistió en el estudio del total de 143 gráficos estadísticos hallados, de los cuales se estudiaron tres variables:

- Tipo de gráfico. Se consideran todos los citados en las directrices curriculares y cualquiera adicional que aparezca en los textos analizados.

- Nivel de complejidad semiótica. Según Arteaga (2011) y Batanero et al. (2010).

- Contexto del gráfico. Según la clasificación en los estudios PISA (OECD, 2019).

Dado que, en relación con cada gráfico, se tiende a proponer más de una actividad, se analizó independientemente cada actividad propuesta para cada gráfico (en total 340 actividades), en las que se estudiaron las siguientes variables adicionales:

Niveles de lectura. Nivel de lectura del gráfico que la ejecución de la actividad requiere por parte del estudiante, considerando los aportes de Curcio y colaboradores (Curcio, 1987; Friel et al., 2001, Shaughnessy et al., 1996).

- Tipo de actividad. Se han considerado leer, completar, calcular, construir, ejemplificar, traducir a otra representación e inventar problema.

- Propósito del gráfico dentro de la actividad. Siguiendo a Kosslyn (1985), hemos considerado análisis, comunicación y meramente constructivo.

En lo que sigue primero se presentan resultados relacionados con la caracterización de los gráficos estadísticos en los textos y luego la caracterización de las tareas.

\section{Caracterización de los gráficos}

A continuación, se presenta el análisis de las variables relacionadas con los gráficos, es decir, tipo de gráfico, contexto de la información y complejidad semiótica del gráfico. 


\subsection{Tipo de gráficos}

La Tabla 1 presenta los resultados al clasificar los gráficos propuestos en los libros de texto según su tipo. Estos resultados muestran que en los libros analizados predominan los gráficos de barras observados en Jiménez-Castro et al. (2020), aunque disminuye su porcentaje $(56,3 \%$ en educación primaria), para dar paso al histograma y polígono de frecuencias, no considerados en la educación primaria y recomendados para $9 .^{\circ}$ curso en las directrices curriculares (MEP, 2012). Aumenta también el porcentaje de gráficos de líneas y sectores (7,2 \% y $9 \%$ en Jiménez-Castro et al., 2020). La presencia del gráfico de barras disminuye por curso, concentrándose el histograma y polígono de frecuencias en 9..$^{\circ}$, sin que aparezcan con anterioridad y según recomendaciones curriculares. Se observan diferencias entre editoriales; SA tiene mayor proporción de gráfico de sectores y PI de gráficos de barras, mientras que PO incluye mayor número de histogramas, gráficos de líneas, barras múltiples y polígonos de frecuencias.

Tabla 1. Distribución (\%) de tipo de gráfico por nivel educativo, editorial y total

\begin{tabular}{|c|c|c|c|c|c|c|c|}
\hline \multirow[b]{2}{*}{ Tipo de gráfico } & \multicolumn{3}{|c|}{ Curso } & \multicolumn{3}{|c|}{ Editorial } & \multirow[b]{2}{*}{$\begin{array}{c}\text { Total } \\
(\mathrm{n}=143)\end{array}$} \\
\hline & $\begin{array}{c}7 .^{\circ} \\
(n=58)\end{array}$ & $\begin{array}{c}8 .^{\circ} \\
(n=44)\end{array}$ & $\begin{array}{c}9 .^{\circ} \\
(\mathrm{n}=41)\end{array}$ & $\begin{array}{c}\mathrm{PI} \\
(\mathrm{n}=30)\end{array}$ & $\begin{array}{c}\mathrm{PO} \\
(\mathrm{n}=45)\end{array}$ & $\begin{array}{c}\mathrm{SA} \\
(\mathrm{n}=68)\end{array}$ & \\
\hline Barras & 43,1 & 31,8 & 12,2 & 40,0 & 22,2 & 32,4 & 30,8 \\
\hline Sectores & 24,1 & 25,0 & 0,0 & 10,0 & 13,3 & 23,5 & 17,5 \\
\hline Histograma & 0,0 & 0,0 & 46,3 & 13,3 & 15,6 & 11,8 & 13,3 \\
\hline Líneas & 8,6 & 18,2 & 4,9 & 13,3 & 13,3 & 7,4 & 10,5 \\
\hline $\begin{array}{l}\text { Polígono de Fre- } \\
\text { cuencias }\end{array}$ & 0,0 & 0,0 & 31,7 & 3,3 & 15,6 & 7,4 & 9,1 \\
\hline Barras Múltiples & 12,1 & 6,8 & 0,0 & 0,0 & 11,1 & 7,4 & 7,0 \\
\hline Diagrama de Puntos & 0,0 & 15,9 & 0,0 & 13,5 & 2,2 & 2,9 & 4,9 \\
\hline Líneas Múltiples & 8,6 & 2,3 & 4,9 & 3,3 & 6,7 & 5,7 & 5,6 \\
\hline Pictogramas & 3,5 & 0,0 & 0,0 & 3,3 & 0,0 & 1,5 & 1,3 \\
\hline
\end{tabular}

\subsection{Complejidad semiótica de los gráficos}

Un elemento que incide en la dificultad de comprensión es la complejidad semiótica del gráfico (Arteaga y Batanero, 2011; Batanero et al., 2010), pues determina el número y dificultad de los conceptos implicados en su interpretación. En el análisis de los textos solo se han encontrado ejemplos de gráficos con complejidad semiótica S2 a S4. Mostramos algunos ejemplos:

S2. Representación de un conjunto de datos uno a uno. No se forma la distribución de datos. Es posible la lectura a nivel 'extracción de datos', al visualizarse todos los valores de las variables, pero no se llega al nivel 'extracción de tendencias'. La Figura 1 es un ejemplo que grafica una lista de datos sin formar su distribución de frecuencias. 

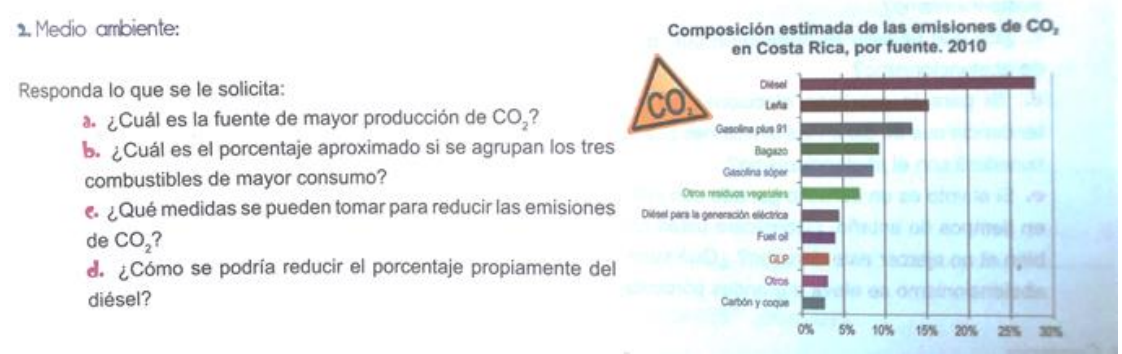

Figura 1. Complejidad semiótica de nivel S2 (PO, 7., p. 103)

S3. Representación de una única distribución de frecuencias. En este nivel se presenta una sola distribución de datos, lo que permite identificar medidas de tendencia central de la variable representada, como moda y mediana. Un ejemplo de este tipo de complejidad semiótica se puede observar en la Figura 2.

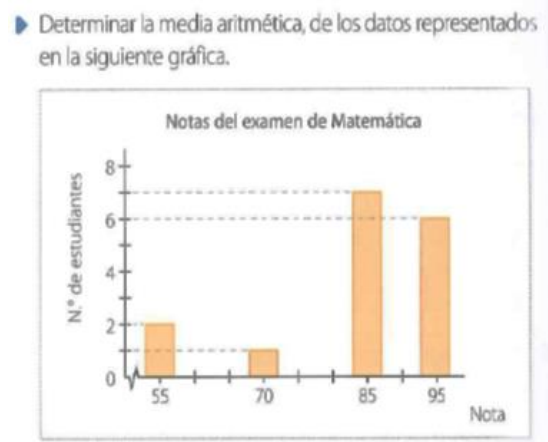

Figura 2. Complejidad semiótica de nivel S3 (SA, $7^{\circ}$, p. 167)

S4. Representación de dos distribuciones sobre un mismo gráfico. Este es el más alto nivel de complejidad semiótica para un gráfico (Arteaga y Batanero, 2011; Batanero et al., 2010). Un ejemplo de este nivel se muestra en la Figura 3, en la que se visualizan dos series de datos que representan los pasatiempos preferidos de niños y adultos. Por tanto, este nivel de complejidad semiótica, además de permitir preguntas sobre cada una de las distribuciones representadas, permite su comparación.

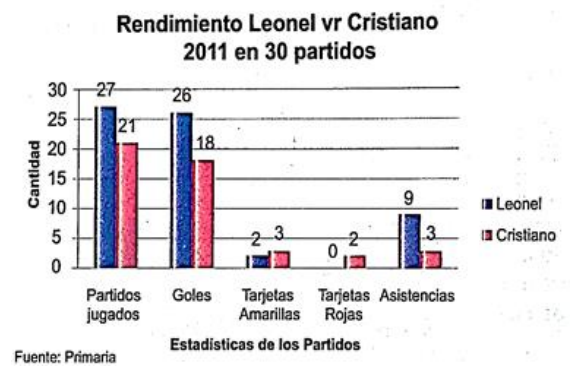

Figura 3. Complejidad semiótica de nivel S4 (PO, $7^{\circ}$, p. 102)

La Tabla 2 presenta los resultados del análisis del nivel de complejidad semiótica de los gráficos. La mayor parte de los gráficos en los libros de texto corresponden al nivel S3, en un porcentaje del $70 \%$ repartiéndose el resto de los gráficos entre los niveles S2 y S4, en proporción similar. Al contrario de lo que cabría esperar, no se obtiene un aumento del nivel de complejidad de los gráficos en los cursos superiores; posiblemente porque al introducir histograma y polígono de frecuencias en el curso noveno, se 
prefiere afianzar estos nuevos gráficos, mediante ejemplos de los niveles S2 y S3. Tampoco se encuentran grandes diferencias en la distribución de esta variable en las tres editoriales.

Tabla 2. Distribución (\%) de nivel de complejidad semiótica de gráficos por nivel educativo, editorial y total

\begin{tabular}{lccccccc}
\hline \multirow{2}{*}{$\begin{array}{l}\text { Complejidad se- } \\
\text { miótica del gráfico }\end{array}$} & $\begin{array}{c}c \\
7 .^{\circ} \\
(\mathrm{n}=58)\end{array}$ & $\begin{array}{c}8 .^{\circ} \\
(\mathrm{n}=44)\end{array}$ & $\begin{array}{c}9 .^{\circ} \\
(\mathrm{n}=41)\end{array}$ & $\begin{array}{c}\text { PI } \\
(\mathrm{n}=30)\end{array}$ & $\begin{array}{c}\text { PO } \\
(\mathrm{n}=45)\end{array}$ & $\begin{array}{c}\text { SA } \\
(\mathrm{n}=68)\end{array}$ & $\begin{array}{c}\text { Total } \\
(\mathrm{n}=143)\end{array}$ \\
\hline S2 & 10,3 & 18,2 & 19,5 & 16,7 & 13,3 & 16,1 & 15,4 \\
S3 & 70,7 & 65,9 & 75,6 & 70,0 & 73,3 & 69,1 & 70,6 \\
S4 & 19,0 & 15,9 & 4,9 & 13,3 & 13,4 & 14,8 & 14,0 \\
\hline
\end{tabular}

\subsection{Contextos de los datos del gráfico}

El contexto es una variable que cobra gran relevancia en educación matemática con las pruebas PISA (OECD, 2019), que tratan de evaluar la competencia del uso de matemáticas de estudiantes en diversos contextos (Wijaya et al., [2014] muestra que el contexto en ocasiones dificulta la resolución de problemas). Ello nos llevó a analizar el contexto de los datos representados en los gráficos, considerando los tipos en PISA (OECD, 2019):

Contexto personal. Situaciones en el ámbito de actividades personales del estudiante, sus amigos, familia o grupo cercano. Ejemplos serían gráficos sobre edades de un grupo de amigos o número de personas en las familias de los compañeros.

Contexto social. Situaciones sociales más amplias que la familia o grupo de amigos, por ejemplo, de la ciudad o la región del alumno, o bien de temas que transcienden lo personal, pero son frecuentes en la vida del alumno. El ejemplo de la Figura 3 se englobaría aquí, pues el alumno con frecuencia ve partidos de futbol y con los amigos discute sobre deportistas. Otros ejemplos en los textos se refieren a distribución de diputados según partido político en el parlamento, tasa histórica de desempleo en el país o bien aprobación de la gestión municipal sobre mantenimiento de áreas verdes.

Contexto laboral o escolar. Situaciones del mundo laboral o la escuela, como calificaciones, empleo, producción, etc. Un ejemplo se presenta en la Figura 2, donde se muestran las calificaciones en matemáticas de un grupo de estudiantes. Otros ejemplos en los textos incluyen datos sobre el promedio de horas de estudio semanal en algunos grupos y distribuciones de género por grados escolares en el centro educativo.

Contexto científico. Situaciones sobre ciencia y tecnología. Ejemplos en los textos son el uso de distintas tecnologías en los hogares, temperaturas máximas y mínimas a lo largo de meses del año, huella de carbono o estudios sobre salud de la población local.

La Tabla 3 resume datos sobre el contexto, donde los contextos están bastante repartidos y en una proporción suficiente cada uno de los cuatro tipos considerados. Al comparar los resultados por curso no se observa una tendencia sistemática, aunque los tipos social y escolar son los más frecuentes en $7 .^{\circ}$ curso, el personal y social en $8 .^{\circ}$ y en $9 .^{\circ}$ curso están igualmente repartidos, salvo el científico con menor proporción en todos los cursos. Hay mayor diferencia en esta variable por editorial. PI tiene tendencia a usar un contexto personal y SA el social. El contexto científico es más frecuente en PO. 
Variables que caracterizan gráficos estadísticos y tareas en libros de texto

Tabla 3. Distribución (\%) de contexto de gráficos por nivel educativo, editorial y total

\begin{tabular}{lccccccc}
\hline \multirow{2}{*}{ Tipo de contexto } & \multicolumn{9}{c}{ Curso } & \multicolumn{5}{c}{ Editorial } \\
\cline { 2 - 8 } & $\begin{array}{c}7 .^{\circ} \\
(\mathrm{n}=58)\end{array}$ & $\begin{array}{c}\mathrm{o}^{\circ} \\
\mathrm{n}=44)\end{array}$ & $\begin{array}{c}9 .^{\circ} \\
(\mathrm{n}=41)\end{array}$ & $\begin{array}{c}\mathrm{PI} \\
(\mathrm{n}=30)\end{array}$ & $\begin{array}{c}\mathrm{PO} \\
(\mathrm{n}=45)\end{array}$ & $\begin{array}{c}\text { SA } \\
(\mathrm{n}=68)\end{array}$ & $\begin{array}{c}\text { Total } \\
(\mathrm{n}=143)\end{array}$ \\
\hline Personal & 12,1 & 29,5 & 26,8 & 53,3 & 13,3 & 13,2 & 21,7 \\
Social & 36,2 & 40,9 & 29,3 & 23,3 & 33,3 & 42,6 & 35,7 \\
Escolar & 32,8 & 15,9 & 24,4 & 13,3 & 28,9 & 27,9 & 25,2 \\
Científico & 19,0 & 13,6 & 19,5 & 10,0 & 24,4 & 16,2 & 17,5 \\
\hline
\end{tabular}

\section{Resultados del análisis de las actividades propuestas para cada gráfico}

Una vez caracterizados los gráficos, pasamos a estudiar las actividades propuestas en relación a los mismos. Para cada actividad de un gráfico, se han analizado el tipo de tarea, el nivel de lectura para llevarla a cabo y el propósito del gráfico en la actividad.

\subsection{Tipo de tarea}

Las tareas de gráficos estadísticos en libros de texto de Costa Rica se clasificaron según categorías de Díaz-Levicoy et al. (2016) y Jiménez-Castro et al. (2020):

Leer el gráfico. Se da un gráfico construido y se solicita obtener información a partir del mismo. Se ve un ejemplo en la Figura 1 donde, dado el gráfico, se plantean preguntas que requieren leer elementos del gráfico para responderlas.

Calcular. Se clasifican las actividades en las que el estudiante, una vez leído el gráfico, debe realizar cálculos con los datos representados. Este tipo se ve en la Figura 2 sobre calcular la media aritmética, a partir de los datos representados. Otras tareas de este tipo piden obtener la moda, máximo o mínimo, y el total de datos en una muestra.

Completar o construir un gráfico. Se completa un gráfico parcialmente construido o bien se construye por completo. Un ejemplo de construcción se ve en la Figura 4, donde el estudiante debe encontrar el máximo y mínimo y determinar los intervalos de clase, para luego determinar la frecuencia en cada clase y elaborar el histograma. El estudiante deberá fijar título, escala y rectángulos que representen la distribución.

15. Construya un histograma a partir de los siguientes datos que corresponden al tiempo,
en minutos, que tardaron 30 estudiantes en terminar su examen de Matemática.
Luego, responda. Defina cinco clases.
\begin{tabular}{|l|l|l|l|l|l|l|l|l|l|}
\hline 42,3 & 39,2 & 58,9 & 42,7 & 38,9 & 39,2 & 70,0 & 67,7 & 45,5 & 69,1 \\
\hline 52,4 & 68,3 & 37,2 & 52,6 & 53,3 & 55,5 & 68,3 & 52,5 & 69,2 & 63,2 \\
\hline 61,9 & 63,9 & 61,2 & 64,9 & 41,9 & 39,2 & 45,7 & 41,7 & 70,1 & 69,8 \\
\hline
\end{tabular}

Figura 4. Construcción de un gráfico (SA, 9. ${ }^{\circ}$, p. 139)

Ejemplificar. Se utiliza el gráfico sólo para efectos de explicar su estructura, para mostrar su utilidad en la extracción de conclusiones o bien para orientar la construcción de un gráfico y sus principales elementos. Un ejemplo se ve en la Figura 3, donde se muestra la utilidad de un gráfico con datos de dos futbolistas para compararlos.

Traducir a otra representación. Se solicita elaborar la tabla de frecuencias que corresponde a los datos representados en un gráfico o bien utilizar otra representación para pasar de un tipo de gráfico a otro. Un ejemplo se ve en la Figura 5, donde el alumno 
debe identificar la variable representada y sus categorías y recordar el concepto de frecuencia, para leer en el gráfico, el porcentaje correspondiente a cada valor y traducirla a la tabla, incorporando igualmente el título y etiquetas de las categorías.

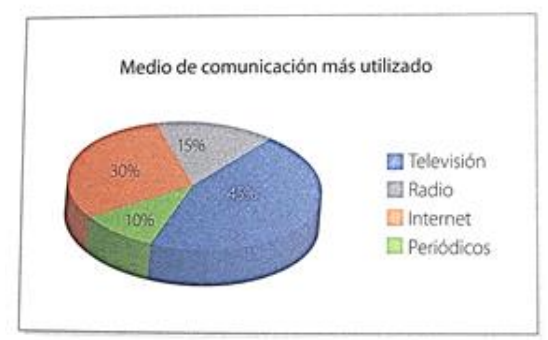

\begin{tabular}{|c|c|c|}
\hline \multicolumn{3}{|c|}{ Medio de comunicación más utilizado } \\
\hline Categoría & $\begin{array}{c}\text { Frecuencia } \\
\text { absoluta }\end{array}$ & $\begin{array}{c}\text { Frecuencia } \\
\text { porcentual }\end{array}$ \\
\hline Televisión & & \\
\hline Periódicos & & \\
\hline Radio & & \\
\hline Internet & & \\
\hline Total & & \\
\hline
\end{tabular}

Figura 5. Traducir a tabla (SA, $8 .^{\circ}$, p. 230)

Inventar problema o extraer conclusiones. Se solicita plantear una nueva situación a partir de los datos de una representación gráfica, o bien extraer una conclusión que se derive de la interpretación de un gráfico. Ejemplos de extraer conclusiones son las dos últimas preguntas de la Figura 1, sobre pensar una conclusión o acción.

Describir una variable. Se pide analizar el tipo de variable representada en una gráfica, como el ejemplo de la Figura 6, donde se debe recordar la definición de variable cuantitativa y que el histograma no es un gráfico adecuado para variables cualitativas.

\section{Histograma}

La gráfica muestra la cantidad de habitantes de un pais, agrupados por edad.

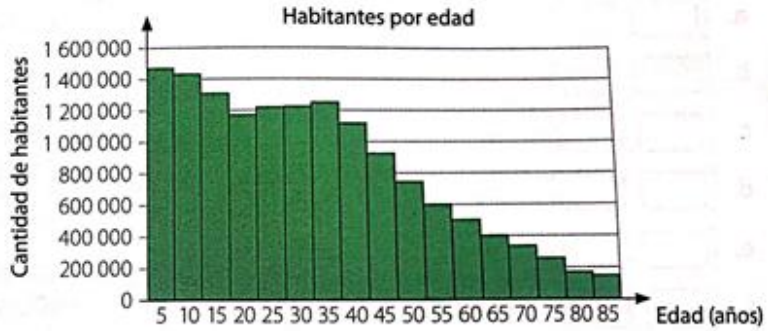

- ¿Qué tipo de variable se representa en la gráfica? Remarque.

Cualitativa Cuantitativa

- ¿Qué diferencias observa entre esta gráfica y una gráfica de barras?

- ¿Qué puede deducir a partir de la información de la gráfica?

Figura 6. Descripción de una variable (SA, 9. ${ }^{\circ}$ p. 136)

Comparar dos representaciones. Se pide comparar la información en dos gráficos, decidir cuál de dos gráficos es preferible para representar unos datos o explicar diferencias entre dos gráficos, como en la segunda pregunta en la tarea de la Figura 6, en que se le pide describir las diferencias entre el gráfico de barras y el histograma.

La Tabla 4 presenta los resultados sobre el tipo de tarea propuesta en relación a los gráficos estadísticos en los textos analizados. Lo más frecuente es proponer actividades de lectura del gráfico, seguidas de tareas de cálculo a partir de los datos del gráfico (lo cual también implicará su lectura). Con menor frecuencia aparece el resto de actividades, añadiéndose dos a las de Díaz-Levicoy et al. (2016): comparar dos representaciones gráficas o describir una variable, ambas con poca frecuencia. 
Las tareas de lectura y cálculo disminuyen con el curso escolar, aumentando las de inventar un problema y en el último curso las de construcción y ejemplificación. Debido a que en este curso se presentan nuevos gráficos y se proponen ejemplos para mostrar el proceso de construcción. Las diferencias entre editoriales son mayores en la invención de problemas y ejemplificación, que se presentan con mayor frecuencia en PO.

Tabla 4. Distribución (\%) de tipo de tarea por nivel educativo, editorial y total

\begin{tabular}{|c|c|c|c|c|c|c|c|}
\hline \multirow[b]{2}{*}{ Tipo de tarea } & \multicolumn{2}{|c|}{ Curso } & \multicolumn{4}{|c|}{ Editorial } & \multirow[b]{2}{*}{$\begin{array}{c}\text { Total } \\
(\mathrm{n}=340)\end{array}$} \\
\hline & $\begin{array}{c}7 .^{\circ} \\
(\mathrm{n}=169)\end{array}$ & $\begin{array}{c}8 .^{\circ} \\
(n=75)\end{array}$ & $\begin{array}{c}9 .^{\circ} \\
(\mathrm{n}=96)\end{array}$ & $\begin{array}{c}\text { PI } \\
(n=109)\end{array}$ & $\begin{array}{c}P O \\
(n=92)\end{array}$ & $\begin{array}{c}\text { SA } \\
(n=139)\end{array}$ & \\
\hline Leer el gráfico & 39,6 & 26,7 & 25,0 & 30,3 & 32,6 & 34,5 & 32,6 \\
\hline Calcular & 31,4 & 28,0 & 22,9 & 37,6 & 13,0 & 30,9 & 28,2 \\
\hline Completar, construir & 4,2 & 24,0 & 16,7 & 13,8 & 12,0 & 10,4 & 12,1 \\
\hline $\begin{array}{l}\text { Inventar problema o extraer } \\
\text { conclusiones }\end{array}$ & 10,7 & 2,7 & 15,6 & 5,5 & 17,4 & 9,4 & 10,3 \\
\hline Ejemplificar & 6,5 & 14,7 & 12,5 & 1,8 & 19,6 & 10,1 & 10,0 \\
\hline Describir variable & 7,1 & 2,7 & 3,1 & 11,0 & 4,3 & 0,7 & 5,0 \\
\hline $\begin{array}{l}\text { Comparar dos representaciones } \\
\text { o traducir a otra representación }\end{array}$ & 0,6 & 1,3 & 4,1 & 0,0 & 1,1 & 3,6 & 1,8 \\
\hline
\end{tabular}

\subsection{Nivel de lectura de los gráficos}

Se analizó el nivel de lectura requerido en las actividades según la clasificación de Curcio (1987), Shaughnessy et al. (1996) y Friel et al. (2001), tomando cuatro niveles que se describieron en los fundamentos y ahora se ejemplifican:

L1. Leer los datos. El nivel más sencillo no precisa comparaciones o cálculos con datos. En la Figura 3 se requiere una lectura literal de cada dato (L1), aunque también se presenta un nivel L2, que se describe para comparar los jugadores. En la Figura 5 se necesita una lectura literal de cada dato del gráfico para construir la tabla.

L2. Leer entre los datos. Se necesita identificar relaciones entre los datos, como ocurre en la pregunta $a$ de la Figura 1 donde la respuesta requiere examinar todos los datos y compararlos para determinar el máximo valor.

L3. Leer más allá de los datos. Se infiere información no representada en el gráfico. La Figura 7 muestra un ejemplo sobre extrapolar un valor en una serie de tiempo.
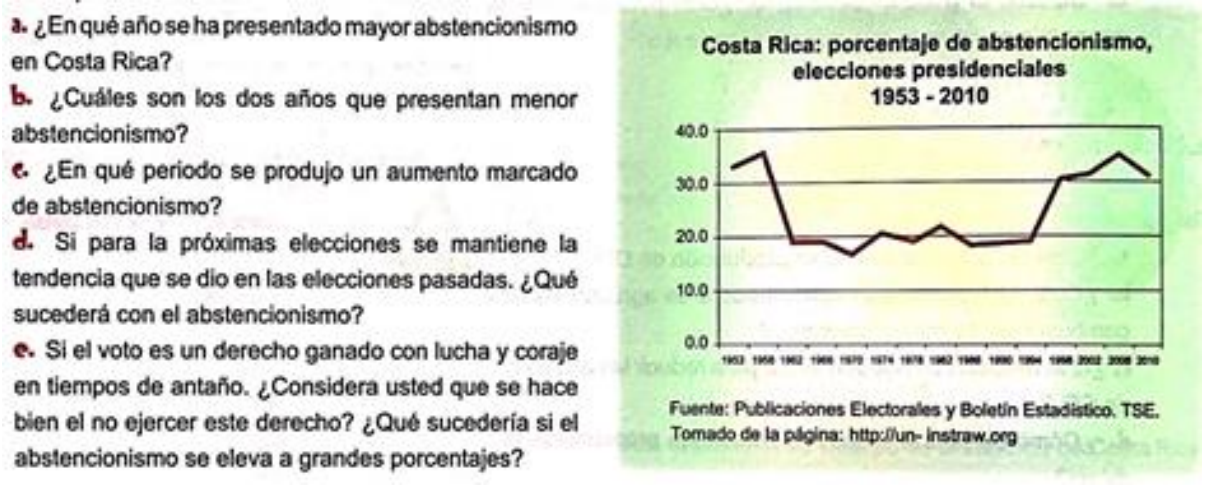

Figura 7. Ejemplo de nivel de lectura 'leer más allá de los datos' 
L4. Leer detrás de los datos. Se realiza una valoración crítica con respecto a las conclusiones realizadas. Un ejemplo son las preguntas c y d de la Figura 1, pues el alumno debe interpretar la información del gráfico y aplicar su conocimiento del tema para proponer algunas soluciones a la problemática planteada.

La Tabla 5 presenta los resultados relativos al nivel de lectura necesario para desarrollar las diferentes actividades propuestas en los textos. El nivel de lectura más frecuente, globalmente, es L2 o 'Leer dentro de los datos', donde se requiere comparar o realizar cálculos con los datos del gráfico, lo que explicamos también por la frecuencia de actividades de cálculo en los libros. Sigue en frecuencia L1 o 'Leer los datos', que solo necesita una lectura literal de valores de la variable o su frecuencia. L3 y L4 que requieren una mayor competencia, aparecen con poca frecuencia. El porcentaje de actividades de L4 o 'Leer detrás de los datos' se incrementa conforme se avanza en el nivel escolar, llegando al 14,6 \% en el 9. ${ }^{\circ}$ curso, donde disminuyen L2 y L3, lo que nos parece adecuado, dada la mayor madurez de los estudiantes. En cuanto a la diferencia entre editoriales, PI presenta más actividades L1 y L2, mientras que PO tiene una distribución más adecuada pues propone con más frecuencia actividades de alto nivel.

Tabla 5. Distribución (\%) de nivel de lectura por nivel educativo, editorial y total

\begin{tabular}{lccccccc}
\hline & \multicolumn{6}{c}{ Curso } & \multicolumn{5}{c}{ Editorial } \\
\cline { 2 - 8 } Nivel de lectura & $\begin{array}{c}{ }^{\circ} \\
(\mathrm{n}=169)\end{array}$ & $\begin{array}{c}{ }^{\circ} \\
(\mathrm{n}=75)\end{array}$ & $\begin{array}{c}9^{\circ} \\
(\mathrm{n}=96)\end{array}$ & $\begin{array}{c}\mathrm{PI} \\
(\mathrm{n}=109)\end{array}$ & $\begin{array}{c}\mathrm{PO} \\
(\mathrm{n}=92)\end{array}$ & $\begin{array}{c}\text { SA } \\
(\mathrm{n}=139)\end{array}$ & $\begin{array}{c}\text { Total } \\
(\mathrm{n}=340)\end{array}$ \\
\hline L1 & 19,5 & 26,7 & 26,0 & 22,0 & 33,7 & 16,5 & 22,9 \\
L2 & 60,4 & 53,3 & 50,0 & 67,9 & 41,3 & 56,1 & 55,9 \\
L3 & 13,6 & 17,3 & 9,4 & 7,3 & 13,0 & 18,0 & 13,2 \\
L4 & 6,5 & 2,7 & 14,6 & 2,8 & 12,0 & 9,4 & 7,9 \\
\hline
\end{tabular}

\subsection{Propósito del gráfico dentro de la tarea}

Kosslyn (1985) considera el gráfico como un recurso que se utiliza para análisis, comunicación y, en la enseñanza tradicional, para fines meramente constructivos. Esta variable sólo se ha tenido en cuenta en nuestro trabajo previo, donde fue una contribución original al análisis de los gráficos en los libros de texto.

Propósito de análisis. El uso principal del gráfico es descubrir o identificar características del conjunto de datos que no son visibles en un listado o tabla de datos. Se trataría de fomentar el proceso de transnumeración, descrito por Wild y Pfannkuch (1999), un proceso básico de razonamiento estadístico. Un ejemplo se ve en la Figura 1, donde se usa el gráfico para descubrir características de las fuentes de emisión de $\mathrm{CO}_{2}$.

Propósito de comunicación. Los gráficos en este tipo se utilizan para transmitir información sobre los datos y relaciones entre ellos. En este caso el constructor del gráfico no precisa descubrir características de los datos, sino comunicarlas a otra persona en forma eficiente. Un ejemplo es la Figura 5 que pide traducir un gráfico a tabla; el propósito del gráfico es comunicar la información requerida para construir la tabla sin que se pida identificar ninguna información a partir del gráfico.

Propósito de construcción. Junto con Kosslyn (1985), añadimos el propósito constructivo, importante desde el punto de vista de la enseñanza tradicional, donde en algunas actividades el interés está centrado en que el estudiante aprenda la construcción del 
gráfico y no en el análisis o la comunicación de información. Un ejemplo se ve en la Figura 4, donde el objetivo es la construcción del gráfico.

La Tabla 6 presenta los resultados del análisis de esta variable. Las actividades analizadas en los libros de texto tienden a utilizar los gráficos con propósito de análisis (46,5 \% del total de actividades), mientras que solo el 15,0 \%, son utilizados con propósito de construcción. Al comparar los resultados por curso, el propósito de análisis aparece con mayor frecuencia en $9 .^{\circ}$ curso, mientras que las actividades de construcción se concentran en los dos últimos cursos. La principal diferencia entre editoriales es que PO realiza más actividades de construcción y menos de comunicación que el resto.

Tabla 6. Distribución (\%) de propósito del gráfico por nivel educativo, editorial y total

\begin{tabular}{|c|c|c|c|c|c|c|c|}
\hline \multirow[b]{2}{*}{ Propósito del gráfico } & \multicolumn{3}{|c|}{ Curso } & \multicolumn{3}{|c|}{ Editorial } & \multirow[b]{2}{*}{$\begin{array}{c}\text { Total } \\
(\mathrm{n}=340)\end{array}$} \\
\hline & $\begin{array}{c}7 .^{\circ} \\
(n=169)\end{array}$ & $\begin{array}{c}8 .^{\circ} \\
(n=75)\end{array}$ & $\begin{array}{c}9 .^{\circ} \\
(\mathrm{n}=96)\end{array}$ & $\begin{array}{c}\text { PI } \\
(n=109)\end{array}$ & $\begin{array}{c}\mathrm{PO} \\
(\mathrm{n}=92)\end{array}$ & $\begin{array}{c}\text { SA } \\
(n=139)\end{array}$ & \\
\hline Análisis & 53,3 & 26,7 & 50,0 & 49,5 & 45,7 & 44,6 & 46,5 \\
\hline Comunicación & 42,6 & 44,0 & 27,1 & 37,6 & 31,5 & 43,9 & 38,5 \\
\hline Construcción & 4,1 & 29,3 & 22,9 & 12,8 & 22,8 & 11,5 & 15,0 \\
\hline
\end{tabular}

\section{Conclusiones}

El objetivo para el trabajo fue caracterizar los gráficos estadísticos en el tercer ciclo de la educación básica (de séptimo a noveno) en Costa Rica y las tareas propuestas en relación a los mismos. Dicho objetivo se ha cumplido para los textos analizados. El análisis de los gráficos estadísticos en los libros para los cursos $7 .^{\circ}$ a $9 .^{\circ}$ en tres editoriales muy utilizadas en Costa Rica complementa la investigación previa en la educación primaria. Pasamos a comentar los principales resultados, a sabiendas de que será necesario ampliar el estudio a otros libros de texto para ver si se replican.

Al igual que en estudios sobre libros de educación primaria, como los de DíazLevicoy et al. (2016) y Jiménez-Castro et al. (2020), en nuestro trabajo se ha encontrado la presencia de gráficos de barras, sectores, líneas, pictogramas y puntos. Además, se incorporan histogramas y polígonos de frecuencia, propios para la representación de variables agrupadas en intervalos, que no se estudian en la educación primaria. Siguen siendo los gráficos de barras los más numerosos, aunque su importancia disminuye en los últimos cursos, para dar paso al histograma y polígono de frecuencia. Aumenta bastante la presencia del gráfico de sectores, especialmente en $7 .^{\circ}$ curso, que apenas tiene presencia en la educación primaria (Jiménez-Castro et al., 2020). En consecuencia, en nuestro trabajo aparecen todos los gráficos sugeridos en el currículo de Costa Rica para la etapa educativa (MEP, 2012), recordándose en $7 .^{\circ}$ curso los estudiados en la educación primaria e introduciendo en $8 .^{\circ}$ y $9 .^{\circ}$ los recomendados en las directrices curriculares.

La complejidad semiótica del gráfico suele ser de nivel S3 o 'Representación de una distribución de datos', lo que también coincide con Díaz-Levicoy et al. (2016) y Jiménez-Castro et al. (2020). Mientras la complejidad semiótica de los gráficos aumentaba en la educación primaria con el curso escolar, en el estudio actual para la educación secundaria no se encuentra diferencia en nivel escolar en los cursos superiores. Pensamos que esto es una debilidad de los libros de texto, que debieran incluir gráficos más complejos en los últimos años de la educación secundaria. 
Respecto al contexto en el que se desarrollan los gráficos, el más frecuente es el social, en contraste con los estudios en educación primaria (Díaz-Levicoy et al.; 2016; Jiménez-Castro et al., 2020) que dan prioridad al contexto personal. El contexto varía conforme se avanza en los niveles educativos, siendo el científico más frecuente en $9 .{ }^{\circ}$ curso y el escolar en $7 .^{\circ}$ curso, lo cual es indicado por la diferente edad de los estudiantes.

La tarea más frecuente en esta etapa educativa es la de leer el gráfico, seguido del cálculo, sin observarse cambios respecto a lo observado en Jiménez-Castro et al. (2020). Sin embargo, aparecen nuevos tipos de tarea, como describir una variable y comparar dos gráficos sin presencia en la educación primaria y que son más adecuadas a la mayor madurez del estudiante y al avance en la formalización del estudio de gráficos. Este tipo de tarea aumenta con el curso, disminuyendo las de lectura y cálculo, lo que supone que el gráfico funciona para mejorar la cultura estadística de los estudiantes.

Los textos analizados hacen uso frecuente de actividades donde se requiere un nivel L2 de lectura o 'Leer dentro de los datos', que suponen más de la mitad de las actividades propuestas. También este nivel fue el más propuesto en la educación primaria, pero ahora incluso aumenta su frecuencia. Los niveles de lectura más altos, L3 y L4, que suponen una lectura crítica, siguen apareciendo con muy poca frecuencia, siendo este un punto a mejorar en la enseñanza de gráficos, pues la lectura crítica es una de las competencias necesarias para alcanzar la cultura estadística, que capacitará a los estudiantes para participar en las sociedades democrática (Engel, 2019).

En cuanto al propósito del gráfico se asume una línea de actividades de análisis y de comunicación, lo cual se alinea con los intereses del Ministerio de Educación Pública de Costa Rica (MEP, 2012) de promover el análisis e interpretación de la información a partir de los datos; también aparecen actividades con propósito constructivo en los dos últimos años donde se introducen nuevos gráficos.

Toda esta información es útil al profesor, que puede tener en cuenta las variables que caracterizan las actividades con gráficos estadísticos y los valores más adecuados para la enseñanza en cada etapa educativa. Hemos citado, por otra parte, diferencias entre editoriales, que pueden incidir en el tipo de presentación de los libros de texto y que el profesor debe considerar a la hora de recomendar uno a sus alumnos.

Finalmente, y puesto que esta investigación versa sobre libros de texto de educación secundaria en Costa Rica, y da continuidad a la realizada en educación primaria en varios países, cabría ampliarla al estudio de gráficos en textos de Bachillerato y universitarios.

\section{Agradecimientos}

Proyecto PID2019-105601GB-I00, AEI y Grupo FQM-126, Junta de Andalucía.

\section{Referencias}

Arteaga, P. (2011). Evaluación de conocimientos sobre gráficos estadísticos y conocimientos didácticos de futuros profesores. Tesis Doctoral sin publicar. Universidad de Granada.

Arteaga, P. y Batanero, C. (2011). Relating graph semiotic complexity to graph comprehension in statistical graphs produced by prospective teachers. En M. Pytlak, T. Rowland y E. Swoboda, Proceedings of the $7^{\text {th }}$ Congress of the European Society for Research in Mathematics Education (pp. 725-734). ERME. 
Batanero, C., Arteaga, P. y Ruiz, B. (2010). Análisis de la complejidad semiótica de los gráficos producidos por futuros profesores de educación primaria en una tarea de comparación de dos variables estadísticas. Enseñanza de las Ciencias, 28(1), 141-154. https://doi.org/10.5565/rev/ensciencias.3627

Curcio, F. R. (1987). Comprehension of mathematical relationships expressed in graphs. Journal for Research in Mathematics Education, 18(5), 382-393. https://doi.org/10.2307/749086

del Pino, J. y Estepa, A. (2019). Análisis de la enseñanza de las medidas de dispersión en los libros de texto. Avances de Investigación en Educación Matemática, 16, 86102. https://doi.org/10.35763/aiem.v0i16.232

Díaz-Levicoy, D., Batanero, C., Arteaga, P. y Gea, M. M. (2016). Gráficos estadísticos en libros de texto de educación primaria: Un estudio comparativo entre España y Chile. Bolema, 30(55), 713-737. https://doi.org/10.1590/1980-4415v30n55a20

Díaz-Levicoy, D., Giacomone, B. y Arteaga, P. (2017). Caracterización de los gráficos estadísticos en libros de texto argentinos del segundo ciclo de educación primaria. Profesorado, 21(3), 299-326.

Díaz-Levicoy, D., Osorio, M., Arteaga, P. y Rodríguez-Alveal, F. (2018). Gráficos estadísticos en libros de texto de matemática de educación primaria en Perú. Bolema, 32(61), 503-525. https://doi.org/10.1590/1980-4415v32n61a10

Engel, J. (2019). Statistical literacy and society. En J. M. Contreras, M. M. Gea, M. M. López-Martín y E. Molina-Portillo (eds.), Actas del Tercer Congreso Internacional Virtual de Educación Estadística. http://www.ugr.es/local/fqm126/civeest.html

Friel, S., Curcio, F. y Bright, G. (2001). Making sense of graphs: Critical factors influencing comprehension and instructional implications. Journal for Research in Mathematics Education, 32(2), 124-158. https://doi.org/10.2307/749671

Gea, M. M., López-Martín, M. M. y Roa, R. (2015). Conflictos semióticos sobre la correlación y regresión en los libros de texto de Bachillerato. Avances de Investigación en Educación Matemática, 8, 29-49. https://doi.org/10.35763/aiem.v1i8.113

Herbel, B. A. (2007). From intended curriculum to written curriculum: Examining the "voice" of a mathematics textbook. Journal for Research in Mathematics Education, 38(4), 344-369.

Jiménez-Castro, M., Arteaga, P. y Batanero, C. (2020). Los gráficos estadísticos en los libros de texto de educación primaria en Costa Rica. Bolema, 34(6), 132-156. https://doi.org/10.1590/1980-4415v34n66a07

Kosslyn, S. M. (1985). Graphics and human information processing. Journal of the American Statistical Association, 80, 499-512. https://doi.org/10.1080/01621459.1985.10478147

Lemos, M. (2006). O estudo do tratamento da informação nos livros didáticos das séries iniciais do Ensino Fundamental. Ciência e Educação, 12(2), 171-184. https://doi.org/10.1590/S1516-73132006000200005

MEP (2012). Programa de estudio matemáticas, I, II y III Ciclos de la educación general básica y ciclo diversificado. Ministerio de Educación de Costa Rica. 
Muñiz, L. J. R. y Díaz, P. (2018). Las investigaciones sobre la estadística y la probabilidad en los libros de texto de Bachillerato.¿Qué se ha hecho y qué se puede hacer? Avances de Investigación en Educación Matemática, 14, 65-81. https://doi.org/10.35763/aiem.v0i14.218

OECD (2019). PISA 2018 mathematics framework. OECD. https://doi.org/10.1787/13c8a22c-en

Porta, L. y Silva, M. (2003). La investigación cualitativa: el análisis de contenido en la investigación educativa. CENDIE.

Reys, B. J., Reys, R. E. y Chavez, O. (2004). Why mathematics textbooks matter. Educational Leadership, 61(5), 61-66.

Roberts, K. L. y Brugar, K. A. (2017). The view from here: Emergence of graphical literacy. Reading Psychology, 38(8), 733-777. https://doi.org/10.1080/02702711.2017.1336661

Shaughnessy, J. M., Garfield, J. B. y Greer, B. (1996). Data handling. En A. J. Bishop, K. Clements, C. Keitel, J. Kilpatrick y C. Laborde (eds.), International handbook of mathematics education (Vol.1, pp. 205-237). Kluwer Academic Publishers. https://doi.org/10.1007/978-94-009-1465-0_8

Spence, R. (1991). Information visualization. Addison-Wesley.

Weiland, T. (2017). Problematizing statistical literacy: An intersection of critical and statistical literacies. Educational Studies in Mathematics, 96(1), 33-47. https://doi.org/10.1007/s10649-017-9764-5

Wijaya, A., van den Heuvel-Panhuizen, M., Doorman, M. y Robitzsch, A. (2014). Difficulties in solving context-based PISA mathematics tasks: An analysis of students' errors. The Mathematics Enthusiast, 11(3), 555-584.

Whitaker, D. y Jacobbe, T. (2017). Students' understanding of bar graphs and histograms: Results from the LOCUS assessments. Journal of Statistics Education, 25(2), 90-102. https://doi.org/10.1080/10691898.2017.1321974

Wild, C. y Pfannkuch, M. (1999). Statistical thinking in empirical enquiry. International Statistical Review, 6, 223-265. https://doi.org/10.1111/j.1751-5823.1999.tb00442.x

\section{Anexo: Textos usados en el análisis}

Publicaciones Innovadoras en Matemáticas para Secundaria (2017). Matemática 7, 8, 9. San José, Costa Rica: Autor.

Publicaciones Porras y Gamboa (2017). Matemática 7, 8, 9. San José, Costa Rica: Autor. Santillana (2017). Matemática 7, 8, 9. San José, Costa Rica: Autor

\section{Referencias de los autores}

Pedro Arteaga, Universidad de Granada (España). parteaga@ugr.es

Maynor Jiménez-Castro, Universidad de Costa Rica. maynorj@correo.ugr.es

Carmen Batanero, Universidad de Granada (España). batanero@ugr.es 


\title{
Variables characterizing statistical graphs and related tasks in Costa Rica secondary education textbooks
}

\author{
Pedro Arteaga, Universidad de Granada \\ Maynor Jiménez-Castro, Universidad de Costa Rica \\ Carmen Batanero, Universidad de Granada
}

Statistical graphs are pervasive in the media and the Internet as a resource for communication and analysis, and given this relevance, the mathematics curriculum in Costa Rica includes its study across Basic Education. The aim of this research was to analyse the variables that characterize the statistical graphs presented in Costa Rica secondary education textbooks. We base on research dealing with analysis of statistical graphs in primary education textbooks, including our work, as well as on studies that analyse the semiotic complexity and reading levels of graphs. Our research is exploratory and qualitative and uses content analysis to describe how the main variables identified in previous research are taken into account in the graphs and related activities in a sample of secondary school textbooks. For each variable, we present the distribution of categories by editorial and by grade level, and compare our results with research carried out with primary education textbooks. Examples of the categories taken from the books analysed are included to make clear the meaning of the category. We first examine 143 graphs presented in three editorials for grades 7 to 9, and study the type of graph, its semiotic complexity, and the context of the data represented. We then study 340 tasks related to these graphs that are included in the textbooks, by analyzing the reading level required, the purpose of the graph within the task, and the type of task. When we compare with results of previous research dealing with primary education, we find that new graphs are introduced, but, still, bar graph have a strong presence. There is a greater presence of the higher level of semiotic complexity (which involves interpretation of the graph content). The school context decreases by giving more weight to scientific context. Regarding the tasks, the weight of reading activities decreases with new activities appearing, such as inventing a problem or describing a variable. The main purpose of graphs is analysis and there is not much difference in the reading level required in primary and secondary education. More research can be conducted by expanding the current body of work into other educational levels and state curricula. 